\title{
lliac Bone Harvesting Techniques for Bone Reconstruction. Comparative Study Between Tricortical Bone Harvesting vs Trapdoor Technique
}

This article was published in the following Dove Press journal:

Therapeutics and Clinical Risk Management

\author{
Jia-Fu Zhu' \\ Wei-Xing $X u^{\prime}$ \\ Qiang $\mathrm{Hu}^{\prime}$ \\ Tian-Quan $\mathrm{Wu}^{2}$ \\ Hong Liu' \\ 'Department of Orthopaedics, Tongde \\ Hospital of Zhejiang Province, Hangzhou \\ 3 I00 I2, People's Republic of China; \\ ${ }^{2}$ Department of Orthopaedics, Shaoxing \\ Keqiao District Hospital of Traditional \\ Chinese Medicine, Shaoxing 312030, \\ People's Republic of China
}

\begin{abstract}
Objective: To investigate the effects of trapdoor-procedure-based bone harvesting and tricortical iliac bone harvesting on the iliac bone-graft donor site pain experienced by patients and their clinical effects.
\end{abstract}

Methods: A retrospective analysis was performed using the clinical data of 65 patients with tibial plateau fractures who received autologous iliac bone-supporting grafts in two hospitals between January 2014 and January 2019. The patients who received trapdoor-procedurebased bone harvesting (34 cases) were in the experimental group, and those who received tricortical iliac bone harvesting (31 cases) were in the control group. This study compared differences in iliac bone-graft donor site incision length, intraoperative blood loss, amount of bones harvested, operation time, and postoperative complications between the two boneharvesting methods. Subsequently, it evaluated the pain experienced by the two patient groups in their iliac bone-graft donor sites and their clinical effects.

Results: One week after surgery, the differences between the iliac bone-graft donor site pain score (measured using SF-MPQ-2) of the experimental group and the control group were not statistically different. However, 3 weeks, 5 weeks, and 3 months after surgery, the iliac bonegraft donor site pain scores of the experimental group were significantly lower than those of the control group. The iliac bone-graft donor site incision length and operation time of the experimental group were not significantly different from those of the control group. However, the iliac bone-graft donor site intraoperative blood loss, amount of bones harvested and the incidence of complications of the experimental group were significantly lower than those of the control group.

Conclusion: Trapdoor-procedure-based bone harvesting has lower donor site pain, intraoperative blood loss, and postoperative complications. However, for bone grafting in regions with significant bone loss, tricortical iliac bone harvesting remains the optimal option.

Keywords: iliac bone, bone grafting, pain, clinical effect

\section{Introduction}

Compared with artificial bone grafting and allogeneic bone grafting, autologous iliac bone grafting has better outcomes in terms of transplant rejection and osteogenesis. ${ }^{1}$ Therefore, autologous iliac bone grafting is commonly used to treat conditions such as bone loss, spondylosis, and joint fusion and is viewed as the gold standard for bone grafting. ${ }^{2,3}$ However, some patients who underwent autologous iliac bone grafting experienced postoperative pain in their iliac bonegraft donor sites and developed other complications. ${ }^{4}$ In this study, a retrospective
Correspondence: Wei-Xing Xu Department of Orthopaedics,Tongde Hospital of Zhejiang Province, 234 Gucui Road, Hangzhou 310012, People's

Republic of China

Tel +86-13750837328

Email xwxspine@।63.com 
analysis was performed using the clinical data of 65 patients with tibial plateau fractures who received autologous iliac bone-supporting grafts (ie, trapdoor-procedurebased bone harvesting or tricortical iliac bone harvesting) in two hospitals (Tongde Hospital of Zhejiang Province and Shaoxing Keqiao District Hospital of Traditional Chinese Medicine) between January 2014 and January 2019 to compare the effects of the two boneharvesting methods on iliac bone-graft donor site pain experienced by the two patients groups and their clinical effects. The results are as follows:

\section{Clinical Data}

\section{Demographic Information}

The study was reviewed and approved by the ethics committee of Medical Ethics Committee of Tongde Hospital of Zhejiang Province and was conducted in accordance with the requirements of the regulatory authorities and with the provisions of the Declaration of Helsinki and the Good Clinical Practice guidelines.Informed consent was exempted, given the retrospective nature of the data collection and the use of deidentified medical records. Personal privacy and individual biological characteristics of patients are strictly confidential. (Theoretical No 2020015). In this study, a retrospective analysis was performed using the clinical data of 65 patients with tibial plateau fractures who received autologous iliac bonesupporting grafts in two hospitals between January 2014 and January 2019. The patients were divided into two groups according to the bone-harvesting method adopted. Those who underwent trapdoor-procedure-based bone harvesting (34 patients) were in the experimental group, and those who underwent tricortical iliac bone harvesting (31 patients) were in the control group. The experimental group consisted of 24 men and 10 women aged between 24 and 62 years (average age: $41.6 \pm 2.7$ years). Of them, 15,11 , and 8 of them had Schatzker II, Schatzker III, and Schatzker IV fractures, respectively. By contrast, the control group comprised 21 men and 10 women aged between 23 and 63 years (average age: $42.2 \pm 2.6$ years). Of them, 13, 10, and 8 had Schatzker II, Schatzker III, and Schatzker IV fractures, respectively. The bone-harvesting operations were performed by the author of this study. The study exclusion criteria were as follows: (a) patients who had a pelvic fracture or bone tumor before surgery; and (b) patients who experienced pain in the pelvis area because of spondylosis. No significant differences were observed between the two patient groups in terms of their ages, genders and Fracture type.which thus made the data suitable for comparison (Table 1).

\section{Surgical Methods}

Trapdoor-Procedure-Based Bone Harvesting

General anaesthesia or spinal anaesthesia was administered.

The hip (donor site) was raised using a pad, and the top of the iliac spine $2 \mathrm{~cm}$ posterior to the anterior superior iliac spine was used to determine the scheduled incision length. A third of the periosteum and fascia at the top of the iliac spine was retained, and the thin plate bone with a thickness of $0.2-0.3 \mathrm{~cm}$ located at said top was cut horizontally (the length of the cut was within the scheduled bone removal length). The thin plate of bone and the periosteum were lifted inward. The bone at the bottom was harvested as needed stopping at the inner table of bone. After harvesting the bones, an absorbable gelatin sponge wrapped with gauzes was used to fill up the bone wound. In situ suture was performed on the thin bone containing the periosteal pedicle. Fasciae in deep layers and those inside the iliac spine were stitched tightly to stop the bleeding completely. Drainage was not needed (Figure 1A-F).

\section{Tricortical lliac Bone Harvesting}

General anaesthesia or spinal anaesthesia was administered. The patient's hip (the donor site) was elevated using a pad, and the top of the iliac spine $(3 \mathrm{~cm}$ posterior to the anterior superior iliac spine) was used to determine the subsequent incision length. A bone scalpel was used to cut off the inner and outer tables of the iliac spine during the surgery. The tricortical bone blocks from the inner table, outer table, and iliac spine were harvested. After the bone harvesting, an absorbable gelatin sponge wrapped with gauzes was used to fill up the bone wound. In the event of excessive bleeding, the bone surface was coated with a thin layer of bone wax to

Table I Preoperative General Conditions of the Two Groups

\begin{tabular}{|l|l|l|l|l|l|l|l|}
\hline \multirow{2}{*}{ Group } & \multicolumn{2}{|l|}{ Gender } & Age & \multicolumn{4}{l|}{$\begin{array}{l}\text { Fracture } \\
\text { Type }\end{array}$} \\
\cline { 2 - 7 } & Male & Female & $(\mathbf{x + s})$ & II & III & IV \\
\hline $\begin{array}{l}\text { Experimental } \\
\text { group } \\
\text { Control group }\end{array}$ & 24 & 10 & $41.6 \pm 2.7$ & 15 & II & 8 \\
\hline $\begin{array}{l}\text { Test value } \\
\mathrm{P}\end{array}$ & 21 & 10 & $42.2 \pm 2.6$ & 13 & 10 & 8 \\
\hline
\end{tabular}


A
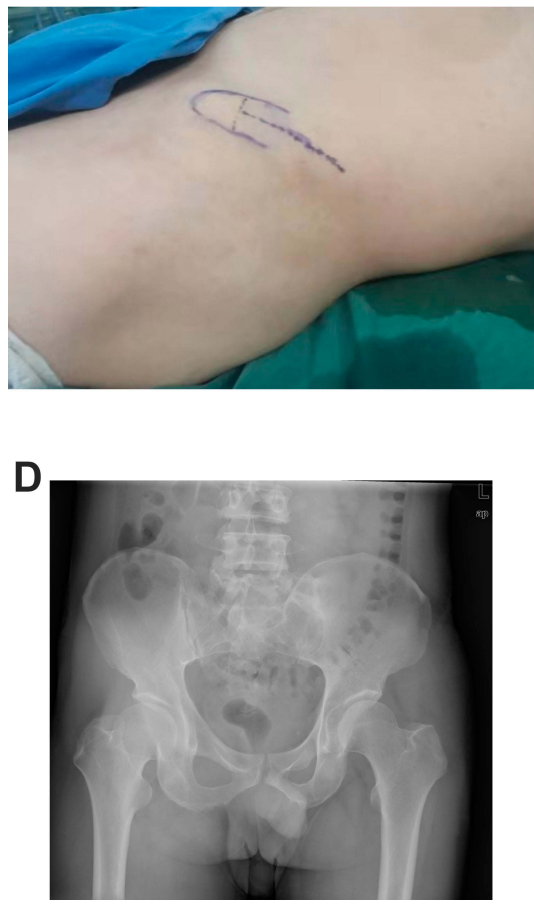

B

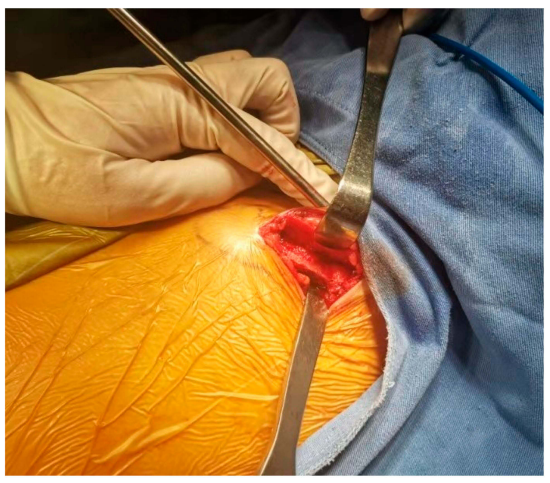

E

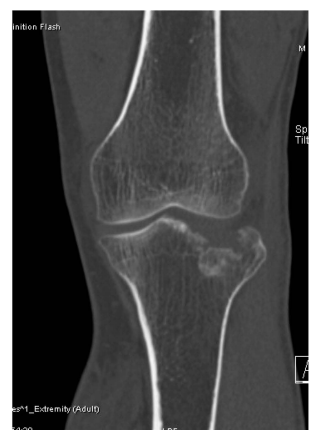

C

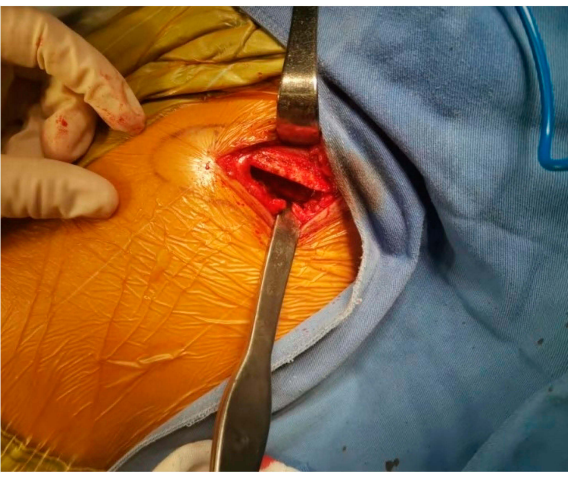

$\mathbf{F}$

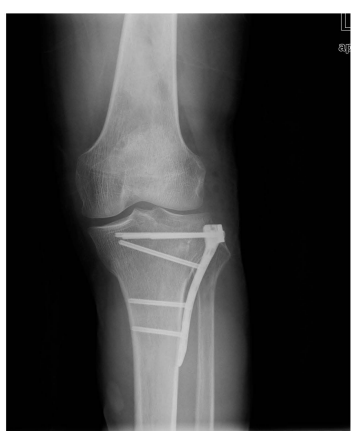

Figure I (A-F) depicts a 45-year-old male patient who was administered general anaesthesia before undergoing open reduction, internal fixation, and bone graft for the treatment of his left tibial plateau fracture. During the surgery, trapdoor-procedure-based bone harvesting was used. (A) illustrates the location of the bone harvesting site prior to surgery. (B) displays the site after the trapdoor was opened. (C) depicts the site when the trapdoor was placed back to the iliac spine. (D) represents a postoperative image of the pelvis. (E) illustrates a clear left tibial plateau fracture and articular surface collapse. (F) illustrates a post-operative left tibial plateau.

seal the gap and stop the bleeding completely. Drainage was not used (Figure $2 \mathrm{~A}-\mathrm{C}$ ).

\section{Postoperative Treatment}

Anti-inflammatory treatment was administered for 24 $\mathrm{h}$ during and after surgery, and patient-controlled analgesia (injected into patients' veins) was offered for 2 days. Painkillers were used or not used for 2 weeks postsurgery according to the requests of patients' painless wards. Anticoagulation was observed for $12 \mathrm{~h}$ postsurgery, and a continuous passive motion machine was used on the third day after surgery to help patients perform functional exercises with their affected limbs. Two weeks after surgery, the patients' stitches were removed, and the patients were discharged from the hospital. None of the patients used painkillers after being discharged from the hospital.

\section{Observation Items and Methods}

The iliac bone-graft donor site pain of the two patient groups was compared 1 week, 3 weeks, 5 weeks, and 3 months after surgery by using the Short-Form McGill Pain Questionnaire 2 (SF-MPQ-2). ${ }^{5}$ The SF-MPQ-2, which comprises four summary scales, can assess continuous, intermittent, neuropathic, and affective pain and is easy to use, saves on time, is reliable, and can comprehensively evaluate neuropathic and nonneuropathic pain. This study compared differences in iliac bone-graft donor site incision length, intraoperative blood loss, amount of bone harvested, operation time, and postoperative complications between the two bone-harvesting methods. Subsequently, this study evaluated the iliac bone-graft donor site pain experienced by the two patient groups and the clinical effects of the bone-harvesting methods.

\section{Statistical Processing}

All data were analyzed using SPSS v19.0. In the beginning of analysis, the data were assessed to determine whether they have a normal distribution. The two patient groups' iliac bone-graft donor site pain scores (measured simultaneously using SF-MPQ-2), incision length, intraoperative blood loss, amount of bones harvested, and operation times were all normally distributed and displayed homogenous variance (represented using $\mathrm{x} \pm \mathrm{s}$ ). Two independent sample $\mathrm{t}$ tests were 


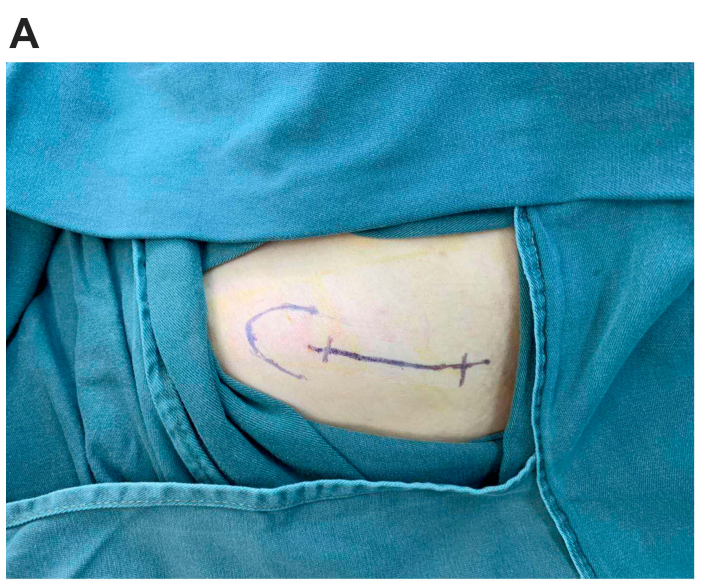

D



B

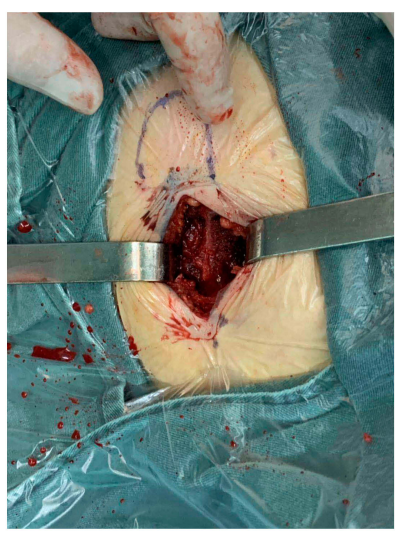

E
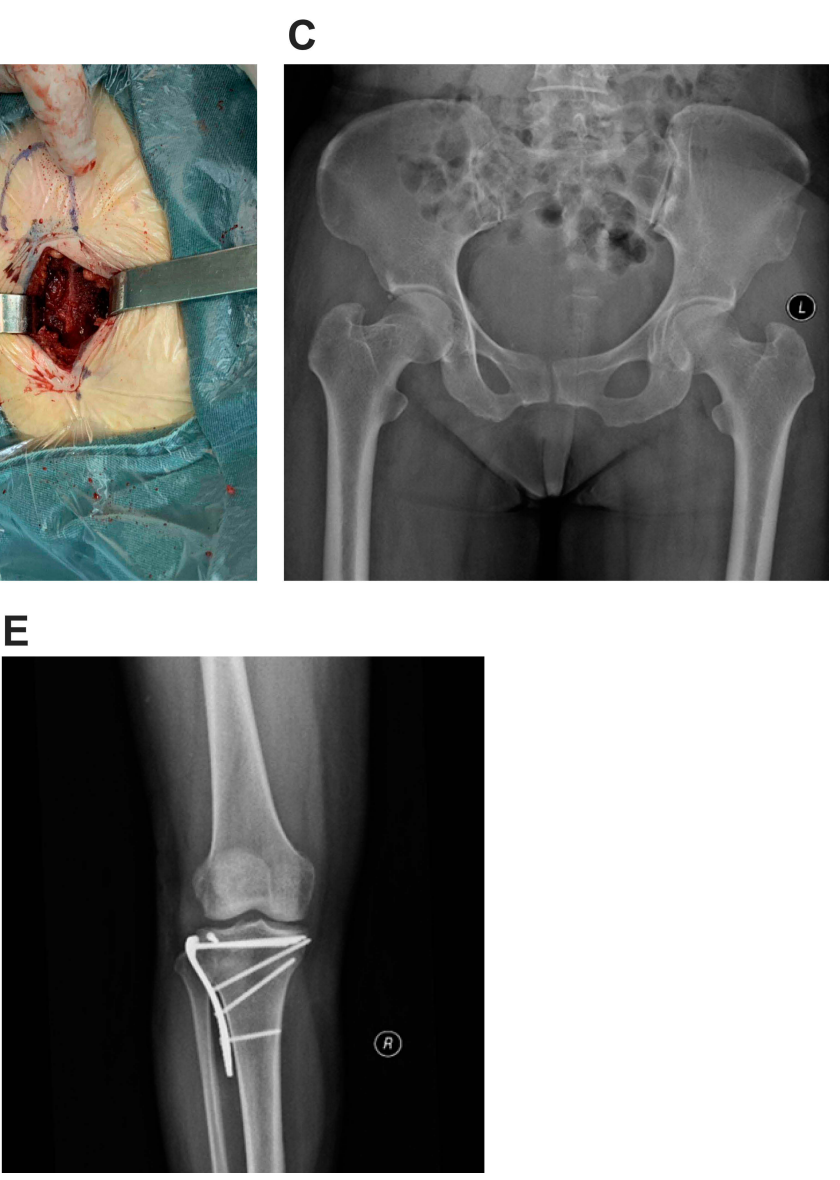

Figure 2 (A-E) depicts a 53-year-old female patient who was administered general anesthesia before undergoing open reduction, internal fixation, and bone grafting to treat her right tibial plateau fracture. During surgery, tricortical iliac bone harvesting was used. (A) illustrates the location of the bone-harvesting site before surgery. (B) displays the bones being harvested. (C) shows a postoperative image of the pelvis. (D) presents a clear right tibial plateau fracture and articular surface collapse. (E) is a postoperative image of the tibial plateau fracture.

then performed. The incidence of complications in iliac bone-graft donor sites for the two patient groups were tested using $\chi^{2}$, where $\mathrm{p}<0.05$ signified statistically significant difference.

\section{Results}

\section{Pain Score Results}

One week after surgery, the iliac bone-graft donor site pain score (measured using SF-MPQ-2) of the experimental group $(6.48 \pm 0.62)$ and that of the control group $(7.21 \pm$ $0.18)$ were compared and were not statistically different
( $p>0.05$ ). However, 3 weeks, 5 weeks, and 3 months after surgery, the iliac bone-graft donor site pain scores of the experimental group $(11.08 \pm 0.45,1.25 \pm 0.03$, and $0.05 \pm$ 0.01 , respectively) were significantly lower $(\mathrm{p}<0.05)$ than those of the control group $(26.45 \pm 0.36,14.54 \pm 0.37$, and $5.64 \pm 0.87$, respectively; Table 2).

\section{Clinical Effects of the Two Bone-Harvesting Methods}

The iliac bone-graft donor site incision length and operation time of the experimental group $(5.18 \pm 0.12 \mathrm{~cm}$ and $12.65 \pm$

Table 2 Iliac Bone-Graft Donor Site Pain Scores (x \pm S; Measured Using SF-MPQ-2) of the Two Patient Groups

\begin{tabular}{|l|l|l|l|l|}
\hline Group & I Week After Surgery & 3 Weeks After Surgery & 5 Weeks After Surgery & 3 Months After Surgery \\
\hline Experimental group & $6.48 \pm 0.62$ & $11.08 \pm 0.45$ & $1.25 \pm 0.03$ & $0.05 \pm 0.01$ \\
Control group & $7.21 \pm 0.18$ & $26.45 \pm 0.36$ & $14.54 \pm 0.37$ & $5.64 \pm 0.87$ \\
Test value & $\mathrm{t}=-1.742$ & $\mathrm{t}=7.257$ & $\mathrm{t}=19.017$ & $\mathrm{t}=23.152$ \\
$\mathrm{P}$ & 0.278 & 0.008 & 0.000 & 0.000 \\
\hline
\end{tabular}


$0.84 \mathrm{~min}$, respectively) were not significantly different $(p>0.05)$ from those of the control group $(5.21 \pm 0.07 \mathrm{~cm}$ and $11.24 \pm 0.67 \mathrm{~min}$, respectively). By contrast, the iliac bone-graft donor site intraoperative blood loss and amount of bones harvested of the experimental group (8.39 \pm $0.62 \mathrm{~mL}$ and $8.35 \pm 0.72 \mathrm{~cm} 3$, respectively) were significantly different $(\mathrm{p}<0.05)$ from those of the control group $\left(21.81 \pm 0.27 \mathrm{~mL}\right.$ and $13.24 \pm 0.27 \mathrm{~cm}^{3}$, respectively). One patient in the experimental group sustained an injury during bone harvesting because of the abnormal course of the lateral femoral cutaneous nerve, which resulted in lateral thigh numbness. The pain in the patient's pelvis area remained after surgery. Four patients in the control group experienced complications during the follow-up period. One patient experienced heterotopic ossification during late-stage hematoma. One patient experienced abdominal wall irritation because of a lumbar hernia. One patient experienced an avulsion fracture of the anterior superior iliac spine (donor site) because of strenuous activity. One patient experienced pain when tying his/her belt because of iliac spine depression. The incidence of complications of the experimental group $(1 / 34)$ was significantly different $(p<0.05)$ from that of the control group (4/31; Table 3).

Figure $1 \mathrm{~A}-\mathrm{F}$ depicts a 45 -year-old male patient who was administered general anaesthesia before undergoing open reduction, internal fixation, and bone graft for the treatment of his left tibial plateau fracture. During the surgery, trapdoor-procedure-based bone harvesting was used. Figure 1A illustrates the location of the bone harvesting site prior to surgery. Figure 1B displays the site after the trapdoor was opened. Figure $1 \mathrm{C}$ depicts the site when the trapdoor was placed back to the iliac spine. Figure 1D represents a postoperative image of the pelvis. Figure 1E illustrates a clear left tibial plateau fracture and articular surface collapse. Figure $1 \mathrm{~F}$ illustrates a post-operative left tibial plateau.

Figure 2A-E depicts a 53-year-old female patient who was administered general anesthesia before undergoing open reduction, internal fixation, and bone grafting to treat her right tibial plateau fracture. During surgery, tricortical iliac bone harvesting was used. Figure 2A illustrates the location of the bone-harvesting site before surgery. Figure 2B displays the bones being harvested. Figure $2 \mathrm{C}$ shows a postoperative image of the pelvis. Figure 2D presents a clear right tibial plateau fracture and articular surface collapse. Figure $2 \mathrm{E}$ is a postoperative image of the tibial plateau fracture.

\section{Discussion}

\section{Characteristics of Autologous lliac Bone Harvesting and Related Complications}

Autologous iliac bone harvesting is a common orthopaedic surgical procedure. Autologous iliac bone grafting can accelerate the anastomosis between the blood vessels and bone grafts in the graft sites, nourish the bone tissues in the graft sites, and induce the generation of new bone tissues, thus facilitating bone healing. ${ }^{6}$ Although iliac bone donor sites are located in a shallow layer, they provide abundant bone supply and facilitate simple bone harvesting operations. Nevertheless, post-operative complications in iliac bone donor sites have been reported. ${ }^{7}$ Less common complications after said surgery include severe events such as peritoneal perforation, urethral injuries, and retroperitoneal hematoma. More common complications after said surgery include nerve damage, hematoma, hernia, ${ }^{8}$ and donor site pain, the last of which is the most prevalent complication. ${ }^{9}$ These complications cause considerable burden on patients' personal and professional life.

\section{Characteristics and Advantages of Trapdoor-Procedure-Based Bone Harvesting}

Currently, trapdoor-procedure-based bone harvesting and tricortical iliac bone harvesting are two common boneharvesting methods. Trapdoor-procedure-based bone

Table 3 Differences in lliac Bone-Graft Donor Site Incision Length, Intraoperative Blood Loss, Amount of Bones Harvested, Operation Time, and Incidence of Complications Between the Two Patient Groups

\begin{tabular}{|l|l|l|l|l|l|}
\hline Group & $\begin{array}{l}\text { Incision } \\
\text { Length } \mathbf{( c m})\end{array}$ & $\begin{array}{l}\text { Intraoperative Blood } \\
\text { Loss } \mathbf{( m L )}\end{array}$ & $\begin{array}{l}\text { Amount of Bones } \\
\left.\text { Harvested } \mathbf{( c m}^{\mathbf{3}}\right)\end{array}$ & $\begin{array}{l}\text { Operation } \\
\text { Time } \mathbf{( m i n )}\end{array}$ & $\begin{array}{l}\text { Incidence of } \\
\text { Complications }\end{array}$ \\
\hline Experimental group & $5.18 \pm 0.12$ & $8.39 \pm 0.62$ & $8.35 \pm 0.72$ & $12.65 \pm 0.84$ & $1 / 34$ \\
Control group & $5.21 \pm 0.07$ & $21.81 \pm 0.27$ & $13.24 \pm 0.27$ & $11.24 \pm 0.67$ & $4 / 31$ \\
Test value & $\mathrm{t}=0.527$ & $\mathrm{t}=6.921$ & $\mathrm{t}=4.258$ & $\mathrm{t}=0.469$ & $\chi^{2}=13.852$ \\
$\mathrm{P}$ & 0.375 & 0.004 & 0.025 & 0.624 & 0.002 \\
\hline
\end{tabular}


harvesting preserves the shape of the iliac spine and prevents iliac bone-graft donor site depression. By contrast, tricortical iliac bone harvesting causes varying degrees of iliac spine damage. The uneven bone edges of iliac bone-graft donor sites irritate the soft tissue, and the pressure created by belt-tying causes pain to the donor site. In addition, removing the inner and outer tables of the bone may cause abdominal hernia ${ }^{10}$ and result in donor site pain. These results supported those of major complications of tricortical iliac bone harvesting from the literature. ${ }^{8}$

Trapdoor-procedure-based bone harvesting preserves the inner tables of the bone and the iliac spine shape. With support from the inner table of the iliac bone and iliac spine, this surgical method enables control of donor site bleeding, limits hematoma, and facilitates the selfremodeling and self-filling of bone wounds during late recovery stages. By contrast, tricortical iliac bone harvesting causes numerous soft tissue peeling in the inner and outer areas of donor sites and cancellous bone exposure, which may increase donor site bleeding. Damage to the donor sites and soft tissue collapses limit control over hematoma, which may potentially spread to locations such as the gluteal and iliacus muscles ${ }^{11}$ and cause heterotopic ossification and pain.

Trapdoor-procedure-based bone harvesting preserves the completeness of the inner table of the bone, which enhances the stability of the inner part of the anterior superior iliac spine and the stretch resistance of the base of the anterior superior iliac spine. As the bones of fractured lower limbs heal, the iliac spine bone also recovers and strengthens the resistance to stretch of the anterior superior iliac spine. By contrast, tricortical iliac bone harvesting leads to poor stability in the base of the anterior superior iliac spine, poor stretch resistance, and increased likeliness of fracture. Among patients who received tricortical iliac bone harvesting in this study, one experienced an avulsion fracture of the anterior superior iliac spine (donor site). For this patient, although the donor site was $3 \mathrm{~cm}$ away from the anterior superior iliac spine, the considerable amount of bones harvested and the bone-harvesting length exceeding $3 \mathrm{~cm}$ still resulted in the patient's poor anterior superior iliac spine stability biomechanically. ${ }^{12,13}$ This revealed that trapdoor-procedure-based bone harvesting remained a safe and effective iliac bone-grafting option despite allowing the harvest of fewer bones than that of tricortical iliac bone harvesting.

\section{Recommendations Regarding Trapdoor-Procedure-Based Bone Harvesting}

(a) Do not penetrate the inner cortex of the trapdoor (ie, the inner cortex of the iliac spine), preserve the completeness of soft issues such as the axial periosteum and fasciae, and induce an anchoring effect to maintain blood flow and facilitate bone reconstruction; (b) If the amount of bones harvested is considerable, multiple trapdoor-procedurebased bone harvesting can be executed to prevent deep bones that are harvested from forming deep-layer hematoma. Harvesting close to the inner tables of bones must be avoided to prevent iliac bone inner table fractures. (c) Damage to the bone harvesting sites can be covered using gauzes and an absorbable gelatin sponge. In case of excessively bleeding, coat the bone surface with a thin layer of bone wax. Nevertheless, this may have a negative effect on donor site reconstruction in late recovery stages.

In sum, trapdoor-procedure-based bone harvesting can significantly lower donor site pain, intraoperative blood loss, and postoperative complications. However, for bone grafting in regions with significant bone loss, tricortical iliac bone harvesting remains the optimal option despite it having a higher incidence of pain in patients' donor sites and more postoperative complications than does trapdoorprocedure-based bone harvesting. Because few patients were studied and the follow-up period was short, more prospective research must be conducted by different medical institutions in the future. In addition, the correlations between related indicators of iliac bone-graft donor sites must be analyzed.

\section{Conclusion}

There was no significant difference in the operation time and incision length between the trapdoor-procedure-based bone harvesting and the tricortical iliac bone harvesting. Trapdoor-procedure-based bone harvesting can significantly lower donor site pain, intraoperative blood loss, and postoperative complications. However, for bone grafting in regions with significant bone loss, tricortical iliac bone harvesting remains the optimal option despite it causing a higher incidence of pain in patients' donor sites and more postoperative complications than trapdoorprocedure-based bone harvesting.

\section{Abbreviation}

SF-MPQ-2, Short-Form McGill Pain Questionnaire 2. 


\section{Data Sharing Statement}

The authors are pleased to share de-identified participant data. The data of this study including figures and tables will be available by contacting corresponding author. The data can be used permanently after the article is published.

\section{Author Contributions}

All authors contributed to data analysis, drafting and revising the article, gave final approval of the version to be published, agreed on the journal to which the article has submitted, and agree to be accountable for all aspects of the work.

\section{Funding}

This study was funded by the Zhejiang Medical and Health Science and Technology Project (project number: 2019KY342).

\section{Disclosure}

All authors declare that they have no conflicts of interest.

\section{References}

1. Li N, Li G, Guan T. Bilateral bone plate with autogenous iliac bone graft in treating Schatzker IV-VI complex tibial plateau fractures. Zhongguo Gu Shang. 2015;28(12):1078-1082.

2. Snavely JE, Mercer RW, Stewart G. Harvest of Iliac crest autograft not associated with localized pain. Int J Spine Surg. 2019;13(3):275-282. doi:10.14444/6037

3. Fucentese SF, Tscholl PM, Sutter R, et al. Bone autografting in medial open wedge high tibial osteotomy results in improved osseous gap healing on computed tomography, but no functional advantage: a prospective, randomised, controlled trial. Knee Surg Sports Traumatol Arthrosc. 2019;27(9):2951-2957. doi:10.1007/s00167-018-5285-8
4. Armaghani SJ, Even JL, Zern EK, et al. The evaluation of donor site pain after harvest of tricortical anterior iliac crest none graft for spinal surgery: a prospective study. Spine (Phila Pa 1976). 2016;41 (4):191-196. doi:10.1097/BRS.0000000000001201

5. Dworkin RH, Turk DC, Revicki DA, et al. Development and initial validation of an expanded and revised version of the short-form McGill Pain Questionnaire (SF-MPQ-2). Pain. 2009;144(1):35-42. doi:10.1016/j.pain.2009.02.007

6. Werle S, AbuNahleh K, Boehm H. Bone morphogenetic protein 7and autologous bone graft in revision surgery for non-union after lumbar interbody fusion. Arch Orthop Trauma Surg. 2016;136(8):10 41-1049. doi:10.1007/s00402-016-2485-x

7. Pan YX, Yang GG, Li ZW, et al. Clinical observation of biomimetic mineralized collagen artificial bone putty for bone reconstruction of calcaneus fracture. Regen Biomater. 2018;5(2):61-67. doi:10.1093/ $\mathrm{rb} / \mathrm{rbx} 033$

8. Shi J, Wang Z, Wang P, et al. Application and comparison of allograft and autograft bone for interbody fusion in cervical tuberculosis. Zhongguo Xiu Fu Chong Jian Wai Ke Za Zhi. 2011;25(11):12 90-1293.

9. Delawi D, Dhert WJA, Castelein René M, et al. The incidence of donor site pain after bone graft harvesting from the posterior iliac crest may be overestimated: a study on spine fracture patients. Spine (Phila Pa 1976). 2007;32(17):1865-1868. doi:10.1097/BRS.0b013e3 $18107674 \mathrm{e}$

10. Malatray M, AI Qahtani T, Monneuse O, et al. Bone and parietal anterior iliac crest reconstruction for trans-iliac hernia after tricortical graft harvesting: an original technique. Orthop Traumatol Surg Res. 2018;104(7):1069-1072. doi:10.1016/j.otsr.2018.06.010

11. Shaw KA, Griffith MS, Shaw VM, et al. Harvesting autogenous cancellous bone graft from the anterior iliac crest. JBJS Essent Surg Tech. 2018;8(3):e20. doi:10.2106/JBJS.ST.17.00068

12. Zermatten $P$, Wettstein $M$. Iliac wing fracture following graft harvesting from the anterior iliac crest: literature review based on a case report. Orthop Traumatol Surg Res. 2012;98(1):114-117. doi:10.10 16/j.otsr.2011.03.026

13. Pirris SM, Nottmeier EW, Kimes S, et al. A retrospective study of iliac crest bone grafting techniques with allograft reconstruction: do patients even know which iliac crest was harvested. J Neurosurg Spine. 2014;21(4):595-600. doi:10.3171/2014.6.SPINE13902
Therapeutics and Clinical Risk Management

\section{Publish your work in this journal}

Therapeutics and Clinical Risk Management is an international, peerreviewed journal of clinical therapeutics and risk management, focusing on concise rapid reporting of clinical studies in all therapeutic areas, outcomes, safety, and programs for the effective, safe, and sustained use of medicines. This journal is indexed on PubMed Central, CAS
EMBase, Scopus and the Elsevier Bibliographic databases. The manuscript management system is completely online and includes a very quick and fair peer-review system, which is all easy to use. Visit http://www.dovepress.com/testimonials.php to read real quotes from published authors. 